\title{
TABLE OF LEGISLATION
}

\section{European Legislation}

\section{Treaties}

Treaty on the Functioning of the European Union (TFEU)

art 101 8.001, 8.008, 8.011, 8.012, 8.018, 8.020, 8.021, 8.025, 8.028

art 101(1)

8.011, 8.040

art 101(1)(a)-(e)

8.011

art 101(3)

8.011, 8.031, 8.040

art 102 8.001, 8.008, 8.011, 8.012, 8.018, 8.020, 8.021, 8.025, 8.028

art 102 (a)-(d)

art 265

8.011

8.040

\section{European Commission and European Parliament Communications}

Payment Services Bill

\begin{tabular}{|c|c|}
\hline para 2.3(b) & 11.012 \\
\hline para 2.4 & 11.008 \\
\hline para 3.9 & $11.021,11.025$ \\
\hline para 3.13 & 11.037 \\
\hline
\end{tabular}

\section{Directives}

Anti-Money Laundering Directive, 5th (MLD5)

2.034, 2.035

Bank Recovery and Resolution Directive 2.013

Capital Requirements Directive (2013/36/ $\mathrm{EU})$

2.013

$\operatorname{art} 3(1)(1)$

3.033

art 8

3.033

art 8(2)

3.034

Database Directive

art 1(2)

art 7(1) art 7(2)(a)

6.009

art 7(5)

6.009

Data Protection Directive (1995) $\quad \mathbf{2 . 0 1 7}$

Deposit Guarantee Schemes Directive 2.013

Directive 2015/849

3.024

Directive (EU) 2015/849

7.003

Directive (EU) 2015/2366 1.004 , 2.002, 3.001, 3.116, 4.002, 5.002, 6.003, 7.001, 12.022

Directive (EU) 2018/843

Directive (EU) 2019/1937

Directive $95 / 46 / \mathrm{EC}$

Directive 96/9/EC

2.034, 7.003

7.004

$6.003,6.004$

Directive 98/26/EC

Directive 2000/46/EC 6.008

4.041

$3.002,7.060$

2.013

Directive 2002/47/EC $\quad \mathbf{2 . 0 1 3}$

Directive 2002/65/EC 1.004, 2.002, 3.001, 4.002, 5.002, 6.003, 7.001, 12.022

Directive 2002/87/EC

Directive 2002/92/EC

Directive 2003/71/EC

$2.013,3.033$

2.013, 7.004

Directive 2004/25/EC

7.111

Directive 2005/56/EC

2.013

Directive 2005/60/EC

Directive 2006/48/EC 7.060

Directive 2006/49/EC

Directive 2007/36/EC

Directive 2007/64/EC

2.013

$3.002,7.060$

2.013, 3.002, 3.033,

$2.013,3.033$

2.013 $3.001,4.002,5.002,6.003,7.001,12.022$

Directive 2009/65/EC

7.106

Directive 2009/110/EC 1.004, 2.002, 3.001, $3.002,3.201,4.002,5.002,6.003,7.060$, 12.022

Directive 2009/138/EC $\quad \mathbf{2 . 0 1 3 , 7 . 0 0 3 , 7 . 1 0 6}$

Directive 82/891/EEC

2.013

Directive 2011/35/EU

2.013

Directive 2011/61/EU

Directive 2012/30/EU

$2.013,7.004,7.106$

2.013 
Directive 2013/36/EU 1.004, 2.002, 2.013, 3.001, 4.002, 5.002, 6.003, 7.001, 7.003, 12.022

Directive 2014/49/EU

Directive 2014/59/EU

Directive 2014/65/EU

Directive 2014/92/EU

Directive 2014/104/EU

Electronic Money Directive, second (EMD2)

3.002, 7.060, 7.083, 7.106, 7.107 art 1(3)

art 2

art 2(2)

art 3(3)

art 4

art 5

art 7(1)

art $7(3)$

art 9

art 10

art 11(4)

art 11(5)

art 11(6)

art $11(7)$

art 12

art 13

3.203

3.118, 3.200

7.069, 7.073

3.032

3.031

3.031

3.206

3.206

3.031, 3.182, 3.203

3.203

3.208

3.208

3.208

3.208

3.209

3.210
Fifth Money Laundering Directive (MLD5)

7.003, 7.012, 7.059

Markets in Financial Instruments Directive (MiFID)

7.004

Markets in Financial Instruments Directive, second (MiFID2)

2.013

Payment Services Directive, second (PSD2)

1.004, 2.002, 2.014, 2.015, 2.016,

2.019, 2.027, 2.051, 3.001, 3.006, 3.010, $3.015,4.002,4.058,5.002,5.014,6.013$, 6.028, 6.029, 6.032, 6.037, 6.042, 6.057, 6.064, 7.001, 7.060, 7.083, 8.014, 8.041, $12.022,12.025$

art 2(1)

art 2(2)

art 2(3)

art 2(4)

art 3

art $3(\mathrm{~b})$

art $3(\mathrm{~h})$

art 4(3)

art 4(8)

art 4(14)

\subsection{2}

3.012

3.012

3.012

3.013, 4.039

3.019

4.039

3.158

3.116, 3.124

4.058 art $4(21)$

3.063

art $4(25)$

7.077

art. 4(29)

2.016

art $4(30)$

3.114

3.148

3.052

3.045

3.023

3.038

3.038

3.032

art 6

art 6(3)

art 6(4)

art 7

arts 7-9

art 8

art 9

art 10

art 14(1)

art 19(1)

art 19(6)

art 19(7)

art 19(8)

art 28

art 29

art 30(2)

art 30(3)

art 31

art 32

art 33(1)

art 34

art 35

art 35(2)

art 36

art 38(1)

art 38(2)

art 40

art 41

art 42

art 43(1)

art 43(2)

art 44

art 45

art 45(1)(b)

art 45(3)

art 46

art 47
$3.027,3.028,3.029$

3.029

$3.031,3.045$

3.044

3.047

3.047

$3.049,3.206$

3.026

3.038, 3.042

$3.189,3.190,3.191$

3.042, 3.043

3.043

3.037, 3.043

3.041

3.041

3.041

3.041

3.030, 3.182

3.035

3.153

3.154, 4.041, 8.054

4.041

3.154, 8.014, 8.055

3.052

3.052

3.053

3.053

3.064, 3.089

3.054

3.054

3.055

3.055

3.012

3.055

3.058

3.058 


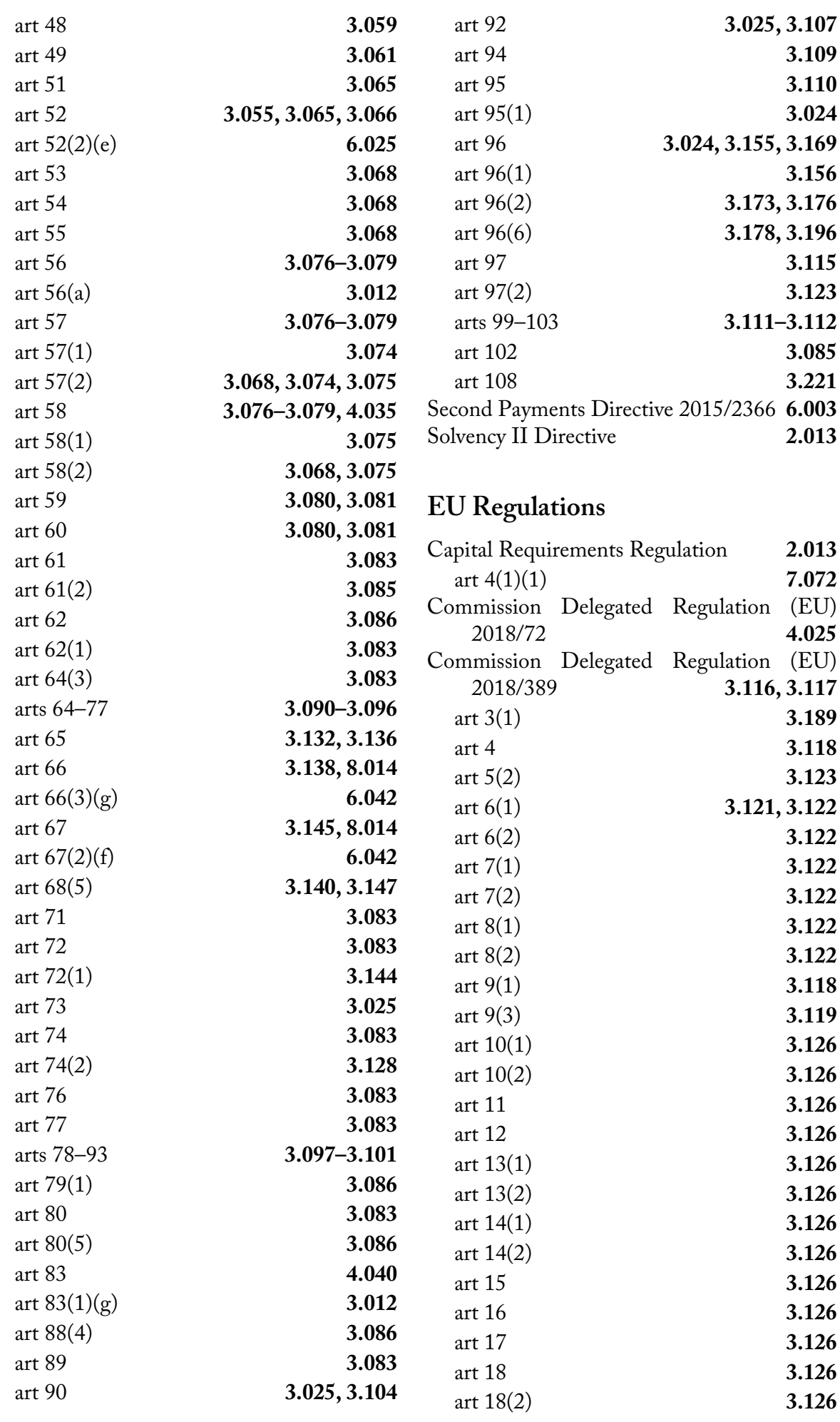




\begin{tabular}{|c|c|c|c|}
\hline $\operatorname{art} 19(1)$ & 3.126 & art 9 & 6.035 \\
\hline art 20(2) & 3.126 & art $9(1)$ & 6.035 \\
\hline art 21(2) & 3.122 & art $9(2)(\mathrm{g})$ & 6.036 \\
\hline $\operatorname{art} 22(4)$ & 3.122 & art 13 & 6.052 \\
\hline art 23 & 3.122 & art 14 & 6.052 \\
\hline $\operatorname{art} 25$ & 3.122 & art 15 & 6.052 \\
\hline art 30(1) & 3.150 & art 17 & 6.052 \\
\hline $\operatorname{art} 30(3)-(5)$ & 3.152 & art 20 & 6.052 \\
\hline art 32 & 3.151 & art 26 & $6.022,6.025$ \\
\hline art 33 & 3.151 & art 32 & 6.056 \\
\hline \multirow{2}{*}{$\begin{array}{l}\text { Council Regulation (EC) No } 1 / 2003 \\
\mathbf{8 . 0 1 6 , 8 . 0 1 8}\end{array}$} & \multirow[t]{2}{*}{ 8.012, } & $\operatorname{art} 32(1)(a)-(d)$ & 6.062 \\
\hline & & art 33 & 6.056 \\
\hline art 3(2) & 8.025 & art 33(1) & 6.066 \\
\hline art 5 & 8.025 & art $34(1)$ & 6.066 \\
\hline art 7 & $3,8.019$ & art $34(3)$ & 6.067 \\
\hline art 9 & 8.019 & art 43 & 6.016 \\
\hline art 11(1) & 8.024 & art 45 & 6.045 \\
\hline art 12 & 8.021 & art 82 & 6.014 \\
\hline art 17 & $8,8.022$ & art $83(5)$ & 6.014 \\
\hline art 18 & 8.018 & art $94(2)$ & 6.028 \\
\hline art 19 & 8.018 & \multirow{2}{*}{\multicolumn{2}{|c|}{$\begin{array}{c}\text { Interchange Fee (Amendment) (EU Exit) } \\
\text { Regulations (2019) }\end{array}$}} \\
\hline $\operatorname{art} 20$ & 8.018 & & \\
\hline art 23 & 8.013 & \multicolumn{2}{|c|}{ Markets in Crypto Assets Regulation (MiCA) } \\
\hline art 23(2) & 8.019 & \multicolumn{2}{|c|}{$7.004,7.060,7.115$} \\
\hline art 31 & 8.020 & art 2(6) & 7.111 \\
\hline art 81 & 8.012 & $\operatorname{art} 3(1)(2)$ & 7.105 \\
\hline art 82 & 8.012 & art $3(1)(22)$ & 7.111 \\
\hline \multicolumn{2}{|c|}{ Cross-Border Payments Regulation, revised } & art 4 & 7.109 \\
\hline (CBPR2) & 2.021 & art $4(2)$ & 7.114 \\
\hline \multicolumn{2}{|c|}{ European Market Infrastructure Regulation } & art 5 & 7.110 \\
\hline & 2.013 & art $19(2)(c)$ & 7.108 \\
\hline Fund Transfers Regulation & 5.008 & art 54 & 7.112 \\
\hline \multirow{2}{*}{\multicolumn{2}{|c|}{$\begin{array}{l}\text { General Data Protection Regulation (2016) } \\
\mathbf{2 . 0 1 7 , 2 . 0 5 3 , 2 . 0 6 0 , 6 . 0 0 3 , ~ 6 . 0 1 1 , ~ 6 . 0 1 4 , ~} \\
\mathbf{6 . 0 1 6 , 6 . 0 2 2 , ~ 6 . 0 2 5 , ~ 6 . 0 2 6 , ~ 6 . 0 3 0 , ~ 6 . 0 3 2 , ~}\end{array}$}} & $\operatorname{art} 58$ & 7.112 \\
\hline & & \multicolumn{2}{|c|}{$\begin{array}{l}\text { Money Laundering and Transfer of Funds } \\
\text { (Information) (Amendment) (EU Exit) }\end{array}$} \\
\hline $6.037,6.043,6.064$ & & Regulations 2019 & 7.003 \\
\hline $\operatorname{art} 4(1)$ & $4,6.014$ & Regulation (EU) 575/2013 & 7.072 \\
\hline $\operatorname{art} 4(7)$ & 6.019 & Regulation (EU) No 648/2012 & 2.013 \\
\hline $\operatorname{art} 4(8)$ & 6.019 & Regulation (EU) No 1093/2010 & $2.013,7.001$ \\
\hline art $4(12)$ & 6.063 & Regulation (EU) 2015/751 & 3.003, 3.088, \\
\hline art 5 & 6.026 & 4.002, 4.026, 4.036, 8.006 & \\
\hline $\operatorname{art} 5(1)$ & 6.041 & Regulation (EU) 2015/847 & $3.024,5.008$ \\
\hline $\operatorname{art} 5(1)(\mathrm{c})$ & 6.038 & Regulation (EU) 2016/679 & $6.003,6.004$ \\
\hline $\operatorname{art} 5(1)(\mathrm{e})$ & & Regulation (EU) 2017/1129 & 7.111 \\
\hline $\operatorname{art} 5(1)(\mathrm{e})$ & 6.040 & Regulation (EU) 2017/2402 & 7.105 \\
\hline art 6 & 6.027 & Regulation 2017, SI 2017/692 & 7.003 \\
\hline $\operatorname{art} 6(1)(a)$ & 6.027 & Regulation 2018, SI 2018/ 1337 & 7.003 \\
\hline $\operatorname{art} 6(1)(b)$ & 6.031 & Regulation (EU) 2021/728 & 4.051 \\
\hline $\operatorname{art} 6(1)(f)$ & 6.031 & Regulation (EC) No 924/2009 & 3.088 \\
\hline
\end{tabular}




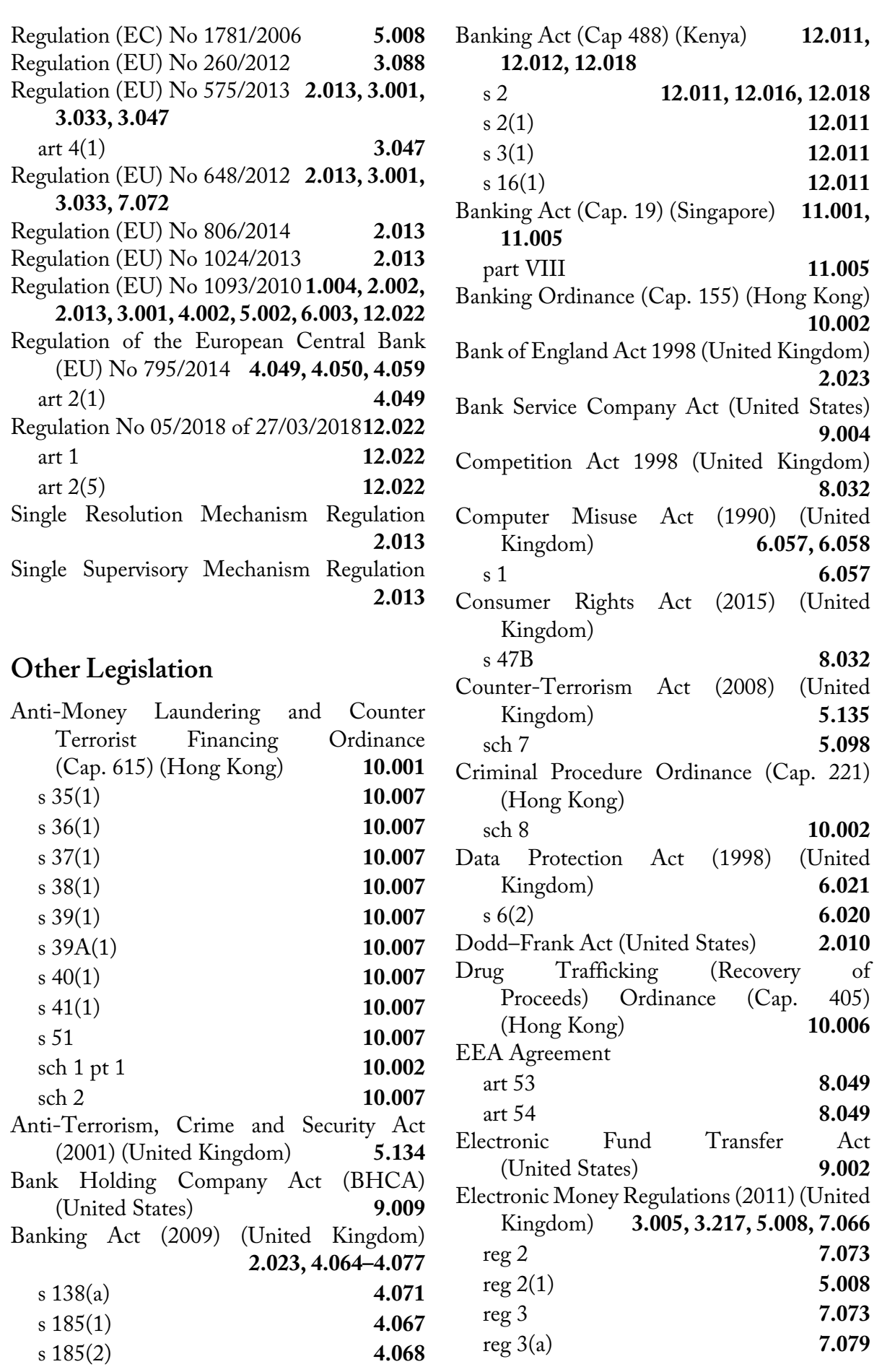




$$
\text { reg } 74
$$

reg $76(1)$

7.066

7.066

European Union (Withdrawal) Act (2018)

(United Kingdom) 3.217, 4.014, 5.008

Financial Services Act (2012) (United Kingdom)

2.023

Financial Services (Banking Reform) Act

(2013) (U.
4.078-4.09
s 42
s 44
s 49
ss $50-53$
s 53
s 104

4.073,

4.080

4.079

4.084

4.082

4.084

s 104

4.085

Financial Services and Markets Act (2000)

(United Kingdom)

$5.008,7.079$

s 19

7.080

s 21(8)(a)

7.088

Part 7

5.008

Financial Services and Markets Act 2000

(Financial Promotion) Order 2005

(United Kingdom)

7.088 sch 1

7.088

Financial Services and Markets Act 2000

(Regulated Activities) Order 2001

(United Kingdom) $\quad \mathbf{7 . 0 6 6}$

Financial Services (Distributed Ledger

Technology Providers) Regulations 2017

(Gibraltar)

Home Owners' Loan Act (United States)

Insurance Ordinance (Cap. 41) (Hong Kong)

9.009
Kong)

10.002

Interchange Fee Regulation (IFR) (United

Kingdom) $\quad 2.063,3.003,4.002,4.003$ art 2(4)

art 2(5)

art 2(6)

art 2(7)

art 2(10)

art 2(11)

art 2(16)

art 2(17)

art 2(18)

art 2(27)

art 2(28)

art 2(31)

art 3

4.008

4.018

4.007

4.019

4.020

4.009

4.009

4.009

4.024

4.024

4.029

4.021

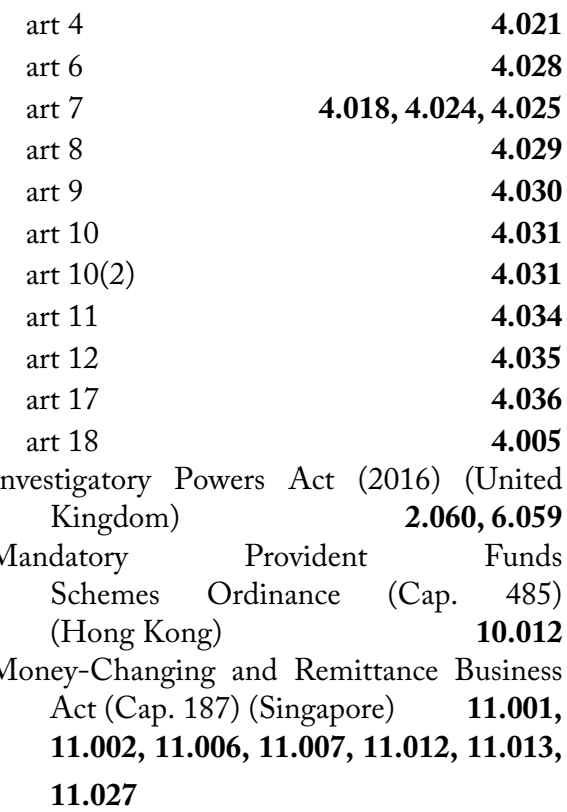

Money Laundering and Terrorist Financing (Amendment) Regulations (2019) (United Kingdom)

2.035

Money Laundering, Terrorist Financing and Transfer of Funds (Information on the Payer) Regulations (2017) (United Kingdom)

5.008

reg 3(1)

5.008

reg 21(5)

5.040

Money Remittance Regulations (2013) (Kenya)

12.015

National Bank Act (NBA) (United States)

9.007

National Payments System Act (2011)

$$
\text { (Kenya) 2.016, 2.017, } 12.015
$$

s 2

12.017

ss $2-6$

12.018

s 12(1)

12.016

National Payments System Regulations (2014) (Kenya) 12.015, 12.020, 12.051 art 2

$12.018,12.020$

art 4

$12.018,12.027$

art 4(1)

12.016

art 5

12.027

art 10

12.030

art 11

12.031

arts $11-18$

12.032

art 15(2)

12.034

art 23(2)

12.034 


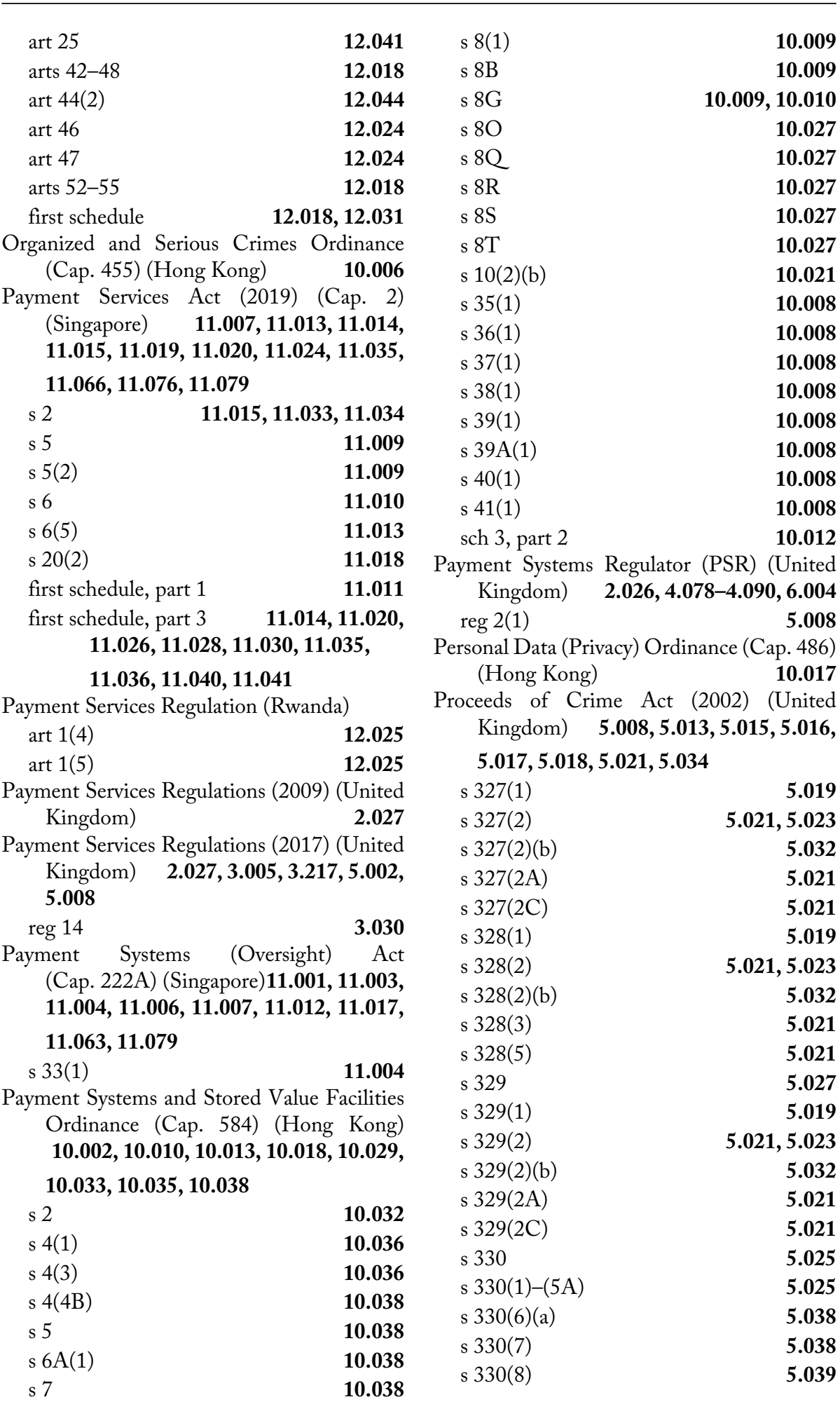




s 331
s 332
s $333 A(1)-(2)$
s $333 A(3)$
s $333 D(3)-(4)$
s 335
s 336A-336D
s 338(1)
s 338(1)-(3)
s 338(2)
s 338(4)
s 338(4A)
s 340
s $340(11)$
s $340(14)$
sch 9 para 1

5.025, 5.040 Regulation of Investigatory Powers Act 5.025, 5.034, 5.040 (2000) (United Kingdom) $\quad \mathbf{6 . 0 5 9}$

5.043 Sanctions and Anti-Money Laundering Act $\mathbf{5 . 0 4 3}$ (2018) (United Kingdom) $\mathbf{5 . 1 3 3}$ 5.043 Securities and Futures Act (Cap. 289) $\mathbf{5 . 0 2 9} \quad \mathbf{1 1 . 0 3 2}$ $\mathbf{5 . 0 2 9} \mathrm{s} 21(\mathrm{a}) \quad \mathbf{9 . 0 4 9}$ 5.029 Securities and Futures Ordinance (Cap. 571) $5.025 \quad \mathbf{1 0 . 0 0 2}$ 5.025 Small Business, Enterprise and Employment $\mathbf{5 . 0 2 9}$ Act (2015) (United Kingdom) $\mathbf{4 . 0 9 0}$ 5.033 Terrorism Act (2000) (United Kingdom) $\mathbf{5 . 0 3 3} \mathbf{5 . 0 0 8}$ 5.016 Truth in Lending Act (United States) $\mathbf{9 . 0 0 2}$ 5.036 United Nations (Anti-Terrorism Measures) 5.021 Ordinance (Cap. 575) (Hong Kong) $\mathbf{5 . 0 1 4} \mathbf{1 0 . 0 0 6}$ Virtual Currency Business Act (2020) (United Kingdom)

$\mathbf{9 . 0 4 6}$ 
John Casanova and Max Savoie - 9781839107986 Downloaded from PubFactory at $04 / 26 / 2023$ 10:43:45AM via free access 